\title{
Clinoidectomía anterior intradural. Estudio anatomo-clínico y utilidad en el tratamiento de los aneurismas paraclinoideos trans-segmentarios C5-C6
}

\author{
J.M. González-Darder
}

Servicio de Neurocirugía. Hospital General de Castellón. Castellón de la Plana

\section{Resumen}

Objetivo. Realizar un estudio anatomoclínico de la clinoidectomía anterior intradural y evaluar su utilidad en el tratamiento microquirúrgico de los aneurismas paraclinoideos. Presentar dos casos de un subtipo de aneurismas paraclinoideos, con el cuello y saco ocupando tanto el espacio clinoideo como el subaracnoideo (aneurismas paraclinoideos trans-segmentarios C5-C6).

Material y métodos. Se ha realizado un estudio microanatómico de la región clinoidea en cráneos secos y espécimenes fijados. Se describen los pasos de la clinoidectomía anterior y los elementos anatómicos que se pueden identificar. Se describen los hallazgos de imagen y quirúrgicos de dos casos de aneurismas paraclinoideos trans-segmentarios C5-C6.

Resultados. El segmento C5 o clinoideo de la carótida interna se convierte en intradural tras la clinoidectomía anterior intradural, con lo que se expone la transición de los segmentos C5 o clinoideo y el C6 u oftálmico, que se moviliza tras la sección del anillo distal de la carótida. Estas maniobras permiten la identificación microquirúrgica del cuello y saco de los aneurismas trans-segmentarios C5-C6 y su exclusión mediante clipaje.

Conclusiones. La nomenclatura de los segmentos de la carótida interna y de los subtipos de aneurismas paraclinoideos permanece confusa y debería unificarse. Las lesiones deberían identificarse por la localización de su cuello (extradural: segmentos C4 y C5; intradural: segmento $\mathrm{C6}$ ), la proyección del saco y donde está la cúpula (intradural o extradural). El clipaje de los aneurismas paraclinoideos se beneficia de la clinoidectomía anterior intradural, que es ineludible para tratar los aneurismas trans-segmentarios C5-C6.

PALABRAS CLAVE: Apófisis clinoides anterior. Clinoidectomía anterior intradural. Arteria carótida interna.

Recibido: 2-06-06. Aceptado: 10-07-06
Aneurismas paraclinoideos. Aneurismas trans-segmentarios C5-C6.

Intradural anterior clinoidectomy. Anatmoclinical study and its usefulness in the tratatment of trans-segmentary C5-C6 trans-segmentary paraclinoid aneurysms

Summary

Ojective. To present an anatomoclinical study of the intradural anterior clinoidectomy and to evaluate the usefulness of this procedure in the microsurgical management of paraclinoid aneurysms. To describe two cases of a subtype of paraclinoid aneurysms arising from and with their fundus growing both in the clinoidal and subarachnoidal segments of the internal carotid artery (paraclinoid trans-segmentary C5-C6 aneurysms).

Material and methods. The microanatomy of the clinodal region has been studied in dried and fixed specimens. The steps of the intradural anterior clinoidectomy are showed. The imaging and microsurgical findings in two cases of trans-segmentary C5-C6 aneurysms are also described.

Results. The C5 clinoid segment of the internal carotid artery is exposed intradurally after anterior intradural clinoidectomy. The transtion between the C5 clinoid and C6 ophthalmic is also exposed and it is movilized only after the section of the carotid distal ring. These manoeuvres allows the microsurgical management and dipping of the trans-segmentary C5-05 aneurysms.

Conclusions. The nomenclature of the internal carotid artery segments and paraclinoid aneurysms remains confuse. Therefore, each lesion should be idetified by the location of the neck (extradural: C4 and C5 segments; intradural: C6 segment), fundus projection and location (intradural / extradural). The microsurgical clipping of the paraclinoid aneurysms is made easier after intradural anterior clinoidectomy, but this manoeuvre is mandatory for trans-segmentary C5-C6 lesions.

KEY WORDS. Anterior clinoid process. Intradural anterior clinoidectomy. Internal carotid artery. Paraclinoid aneu- 


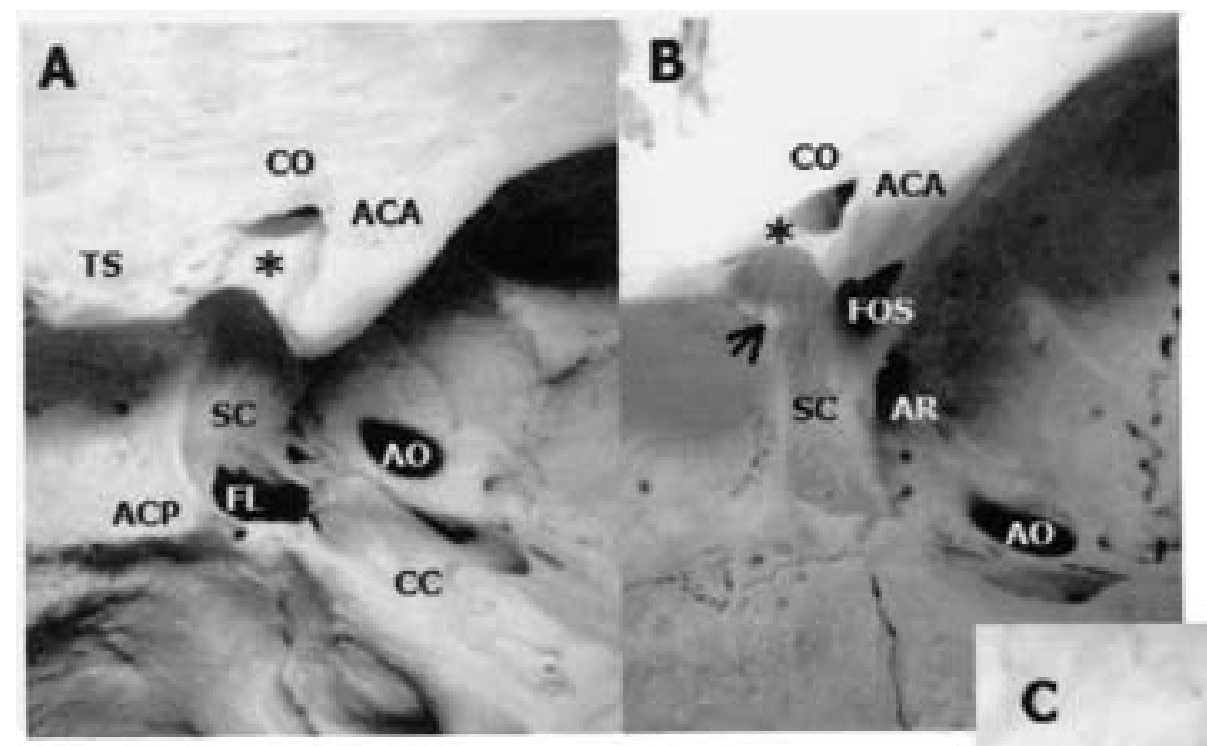

Figura 1. A: Visión superior de la región de la apófisis clinoides anterior (ACA) en un cráneo seco, donde se aprecian la clinoides posterior (ACP) y el tuberculum sellae (TS). La carótida seguiría el canal carotídeo (CC), agujero rasgado anterior o foramen lacerum (FL) y el surco carotídeo (SC) hacia el "optic strut" (*). Se aprecian dos orificios, el canal óptico (CO) para el IIp y el agujero oval (AO) para V3. B: En una visión mas posterior se destaca la apófisis clinoides media (flecha) y se visualizan la hendidura esfenoidal o fisura orbitaria superior (FOS) y el agujero redondo mayor (AR) para el V2. C: Visión intraorbitaria del fondo de la órbita, donde se aprecia el "optic strut" (*) entre el canal óptico (CO) y la fisura orbitaria superior (FOS).

rysms. Trans-segmentary C5-C6 aneurysms.

\section{Introducción}

En los últimos años el desarrollo de las técnicas de estudio microneuroquirúrgico en espécimenes anatómicos especialmente preparados con métodos de fijación sofisticados e inyectados con silicona de colores ha permitido un gran avance en el conocimiento del seno cavernoso y en el tratamiento de las lesiones relacionadas con el mismo. El interés sobre la intrincada anatomía del seno cavernoso comienza con Dwight Parkinson, pero pronto aparecen las figuras de Vinko Dolenc y la de Albert Rothon que lo estudian exhaustivamente, permitiendo el abordaje extradural o intradural al mismo ${ }^{4,13,14}$. Al tiempo se desarrollan abordajes quirúrgicos que permiten exposiciones muy amplias a la zona sin apenas retracción cerebral y muy bien toleradas por los pacientes.

En la actividad quirúrgica rutinaria no se precisa utilizar para cada paciente todas las posibilidades de este tipo de abordajes anatómicos, que deben ajustarse a cada caso

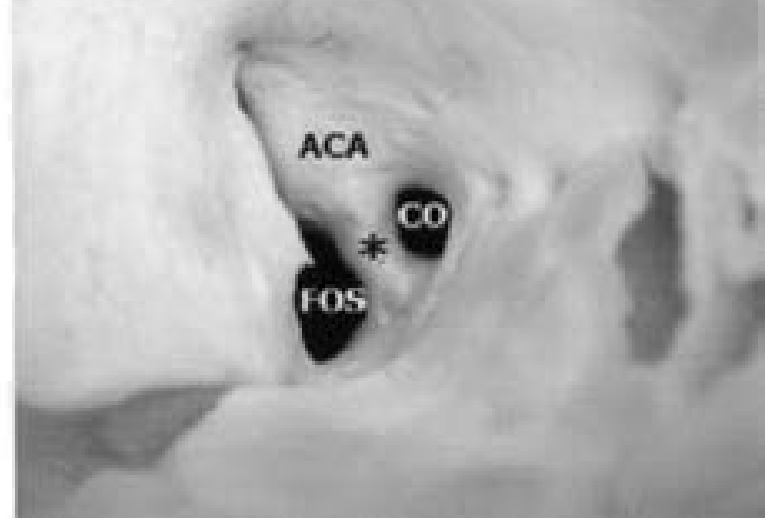

concreto. En este sentido, probablemente la clinoidectomía anterior intradural es uno de los recursos quirúrgicos de mayor utilidad práctica, ya que permite exponer el segmento clinoideo de la arteria carótida interna. En este trabajo describiremos los detalles anatómicos y técnicos de la clinoidectomía intradural y su utilidad en el tratamiento de uno de los subtipos de aneurismas paraclinoideos, los que se originan en la transición de los segmentos clinoideo y oftálmico de la arteria carótida interna.

\section{Anatomía microquirúrgica}

Hemos utilizado un total de seis espécimenes anatómicos obtenidos de necropsia, fijados con formol diluido y sin inyección de colorantes, así como cuatro cráneos secos. En los espécimenes se realizó una clinoidectomía anterior intradural utilizando el mismo material que en la cirugía convencional (microscopio quirúrgico Leica Wild 695, motor Midas Rex 3 con fresas de diamante, material de microcirugía) y se tomaron fotografías con una cámara digital Soni Cyber-shot DSCT1. 

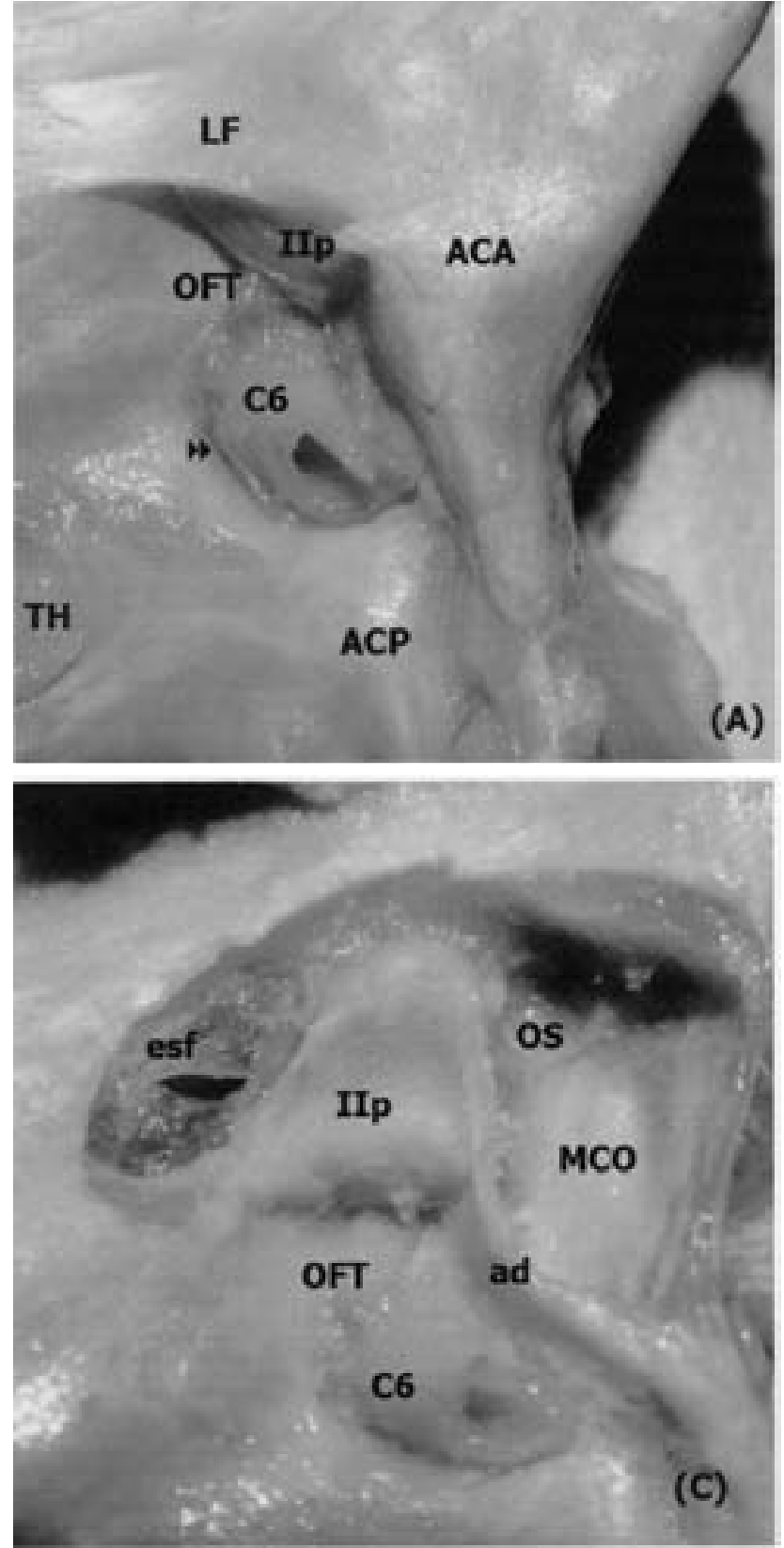

Figura 2. Clinoidectomía anterior intradural. A: Se aprecian los elementos óseos cubiertos por la duramadre, la apófisis clinoides anterior (ACA) y posterior (ACP). El ligamento falciforme (LF) recubre el nervio óptico (IIp) y debajo se aprecia el segmento C6 de la arteria carótida interna con la arteria oftálmica (OFT) emergiendo de la misma. Se ve el tallo hipofisario seccionado (TH) y el "carotid cave" (•). B: Aspecto tras la resección de la duramadre que cubre el canal óptico y apófisis clinoides anterior, conservando el ligamento falciforme (LF). C: Tras la resección del ligamento falciforme y el destechado del canal óptico se expone el nervio óptico (IIp) y la arteria oftálmica (OFT). Por dentro se ha fresado el hueso para exponer la mucosa del seno
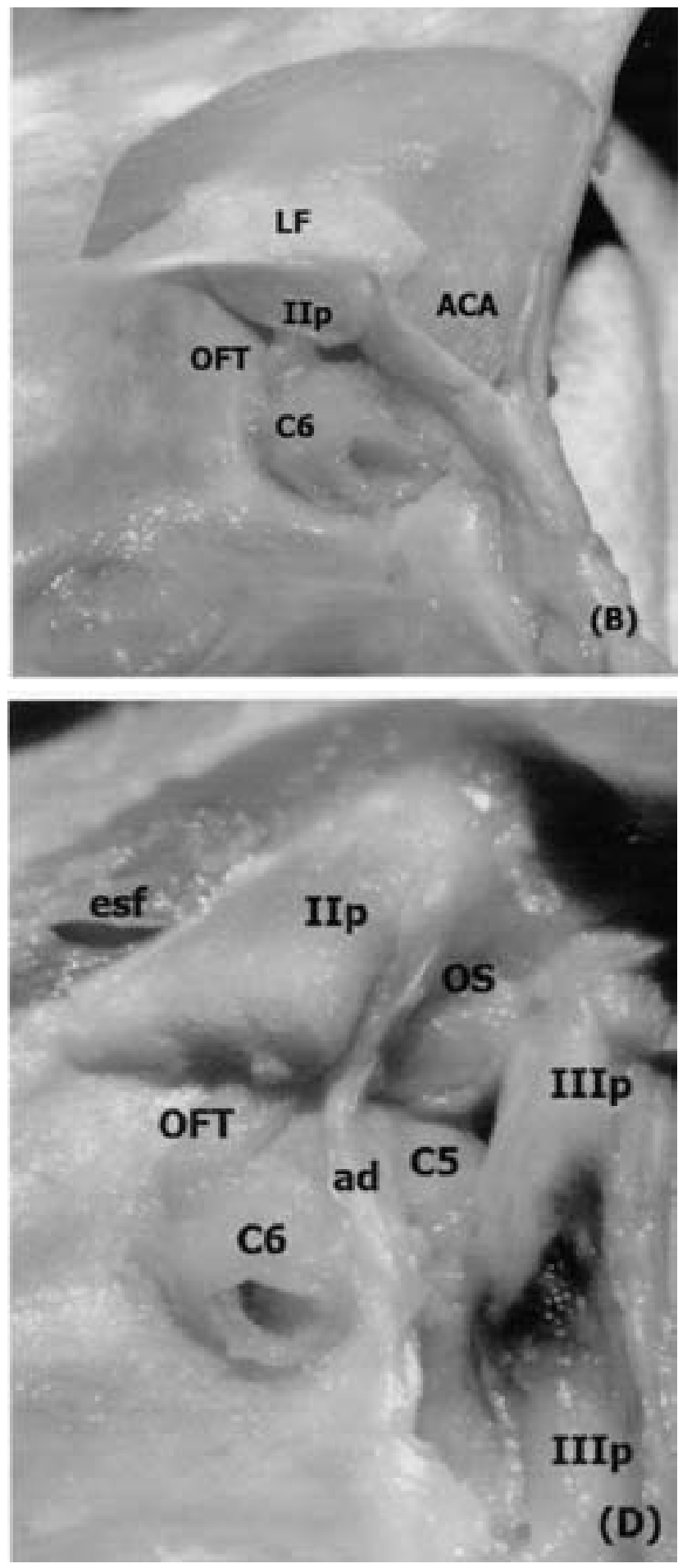

esfenoidal (esf). La resección de la clinoides permite exponer la membrana carotido-oculomotora (MCO) y el muñón del "optic strut" (OS), así como el anillo dural o distal (ad). D: Tras extirpar la membrana carotido-oculomotora se reconoce el segmento C5 de la arteria carótida interna y el trayecto del nervio oculomotor (IIIp). El anillo distal (ad) separa los segmentos C5 y C6. 


\section{Apófisis clinoides anterior}

En el cráneo seco (Figura 1) visto desde su vertiente craneal la apófisis clinoides anterior (ACA) se proyecta en sentido posterior y se le reconoce una forma de pirámide triangular. Sus caras superior y externa son continuación del ala menor del esfenoides y la base se continúa con el techo de la órbita y ala del esfenoides. La cara medial es mas compleja, ya que proximalmente forma la parte externa del canal óptico (CO) y más distalmente presenta un puente o arbotante óseo que llega al cuerpo del esfenoides, denominado "optic strut" (OS). Este puente óseo es fácilmente visible a través de la órbita. Pueden haber ocasionalmente otros dos puentes óseos desde la punta de la ACA, uno hacia la clinoides posterior (ACP) y otro hacia la llamada clinoides media.

En el cráneo con las partes blandas (Figura 2 A) la duramadre recubre los elementos óseos y se encuentran una serie de pliegues: interclinoideo, petroclinoideo anterior y petroclinoideo posterior. La dura de la fosa anterior cubre parcialmente el nervio óptico formando el ligamento falciforme (LF) y a la silla turca ocupada por la hipófisis, dejando un orificio para la salida del tallo hipofisario o diafragma sellar. El nervio óptico (IIp) penetra en el agujero y canal óptico debajo del LF, sobre el OS y por dentro de la ACA. La arteria carótida interna (ACI) en su segmento C6 u oftálmico se ve emerger por debajo y fuera del IIp atravesando una membrana dural que recubre el plano óseo y que se conoce como anillo distal (ad), dural o fibroso. Si se moviliza la ACI lateral o medialmente es posible descubrir un espacio virtual entre la misma y la duramadre, mientras que en los espécimenes anatómicos estudiados, al tener el nervio óptico seccionado, es posible ver la salida de la arteria oftálmica (OFT), ya que generalmente se origina en el tercio medial de la ACI distalmente al anillo distal y se coloca por debajo del IIp. El espacio virtual situado ventromedialmente a la ACI se conoce como "carotid cave". Por detrás del final aparente de la ACA se aprecia la entrada del IIIp en el seno cavernoso.

\section{Clinoidectomía anterior intradural}

Se inicia realizando un amplio corte arqueado de la duramadre del techo de la órbita y ala del esfenoides, rebatiéndola sobre el nervio óptico y carótida interna. Se secciona la duramadre y se reseca el LF para exponer todo el hueso (Figura 2 B). Inicialmente se expone el nervio óptico fresando el techo del canal óptico. La ACA puede extirparse de dos formas. Puede resecarse en bloque fresando transversalmente por su base a nivel de canal óptico previamente destechado, desconectándola del techo de la órbita y del ala del esfenoides. Se despega luego de la duramadre que la envuelve y se rompe o fresa finalmente el OS, que es el último elemento que la retiene. Alternativamente puede fresarse la cortical de toda la cara superior de la ACA y la esponjosa de la misma hasta ahuecarla completamente, incluyendo el OS. Se separa ahora la duramadre y se va extirpando poco a poco la cortical. En el destechado del canal óptico se ha de ser cuidadoso, ya que medialmente al IIp se accede a la mucosa del seno esfenoidal (ESF), separada del espacio intracraneal por una finísima capa de hueso.

Los elementos que se exponen son los siguientes (Figuras $2 \mathrm{C}$ y $2 \mathrm{D}$ ). La ACI intradural en su segmento $\mathrm{C} 6 \mathrm{u}$ oftálmico es la continuación del segmento C5 o clinoideo, quedando ahora separados por el anillo distal. El segmento clinoideo mide no más de $5 \mathrm{~mm}$ de longitud y describe una curva hacia abajo y afuera. Por delante se aprecia el

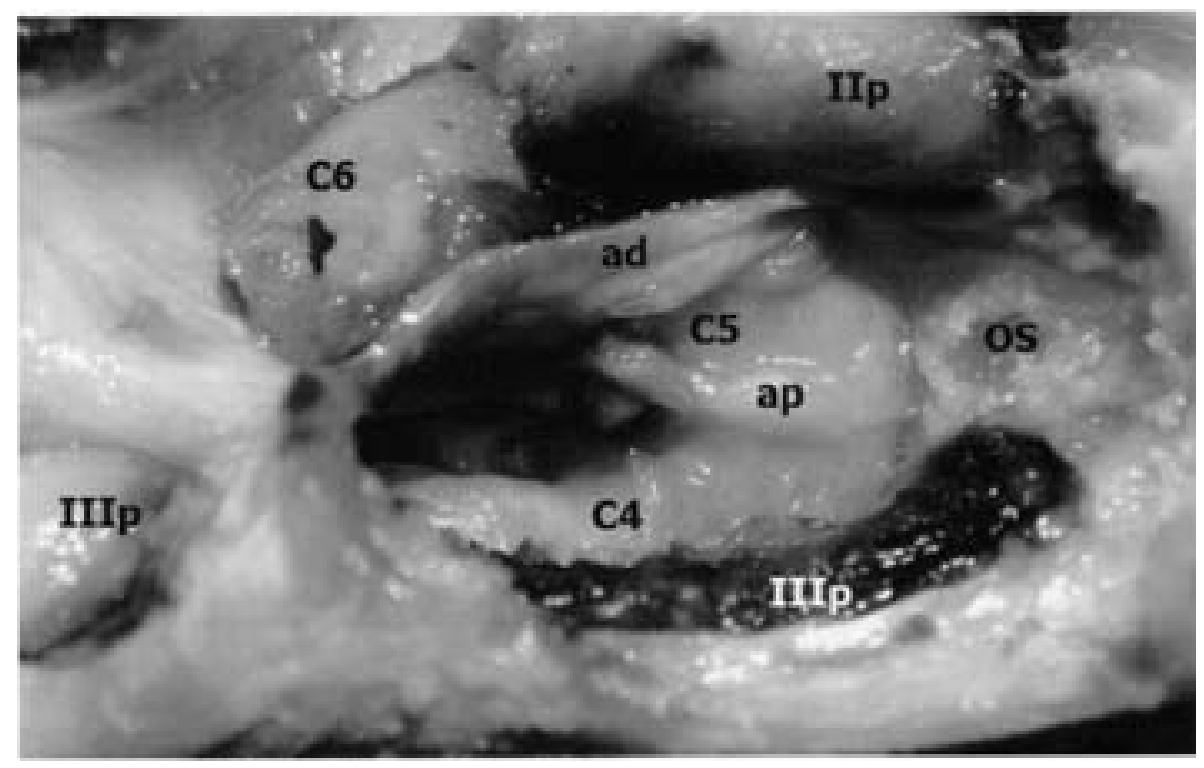

Figura 3. Visión lateral tras clinoidectomía anterior. Se reconocen el anillo distal (ad) que separa los segmentos C5 y C6 y el anillo proximal (ap) que separa los segmentos $C 4$ y C5. La porción clinoidea C5 de la arteria carótida interna se delimita entre ambos anillos y se relaciona con el "optic strut" (OS). 

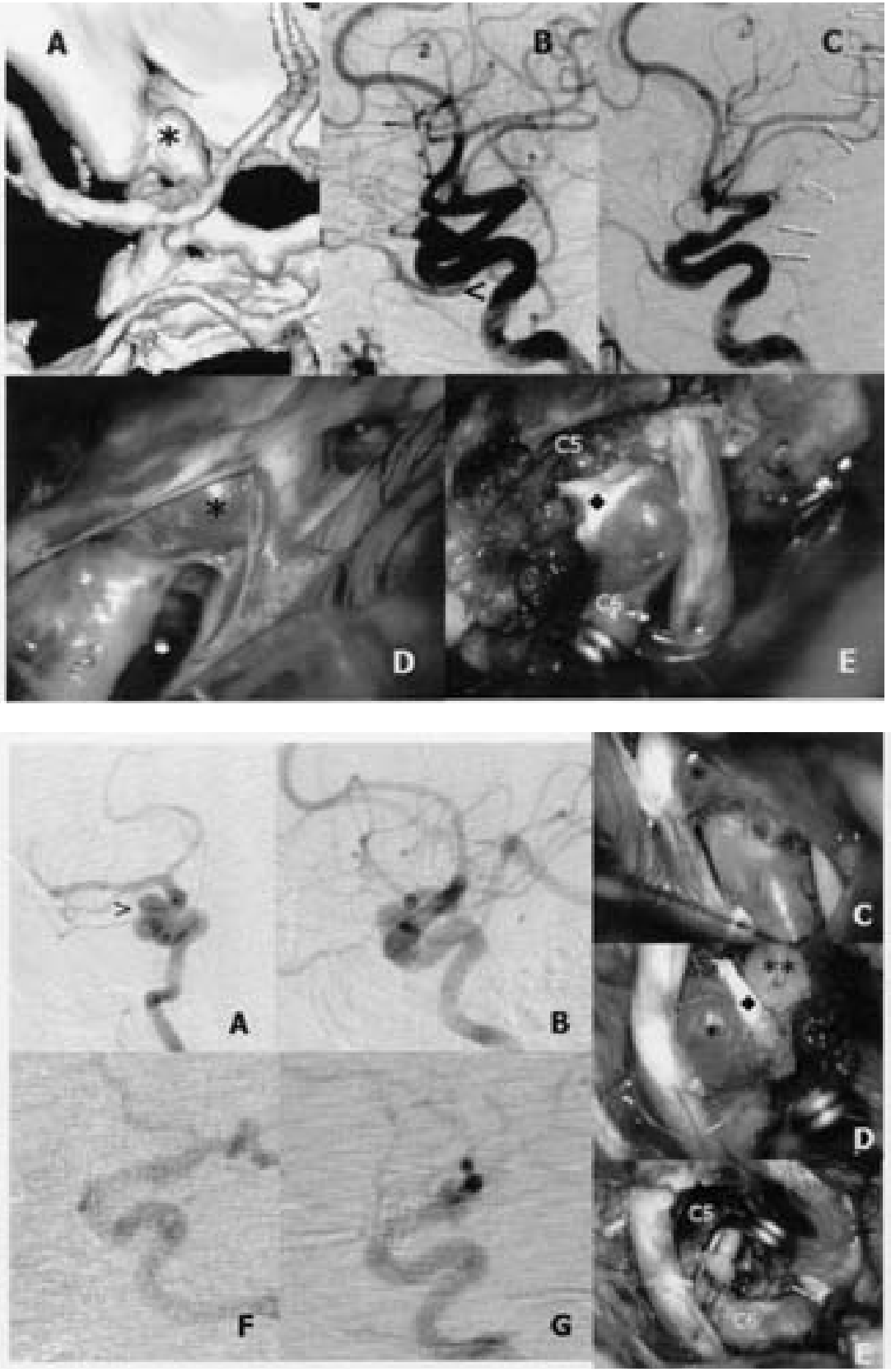

Figura 4. Caso 1. A: Angio-TAC con reconstrucción $3 D$, donde se visualiza la porción intradural del aneurisma (*) por dentro de la apófisis clinoides. B: Angiografía lateral preoperatoria mostrando un aneurisma paraclinoideo izquierdo de proyección superior. Obsérvese el origen de la arteria oftálmica en el segmento intracavemoso C4 de la carótida interna (<). C: Angiografía postoperatoria tras el clipaje y exclusión del aneurisma. $D$ : Fotografía intraoperatoria de la lesión antes de la clinoidectomía. E: Fotografia intraoperatoria tras la clinoidectomía, donde se aprecia la porción cliniodea (C5) y oftálmica (C6) de la carótida interna y como el cuello del aneurisma, que se extiende a lo largo de ambos segmentos, separados por el anillo distal (•).

Figura 5. Caso 2. A y B: Angiografia oblicua y lateral preoperatoria mostrando un aneurisma paraclinoideo izquierdo de proyección superior y de aspecto bilobulado por una indentación (>). C: Fotografía intraoperatoria de la lesión (*) antes de la clinoidectomía. D: Fotografía intraoperatoria tras la clinoidectomía, donde se aprecia las porciones intradural ${ }^{*}$ y clinoidea $(* *)$ del aneurisma, separadas por el anillo distal (๑). E: Fotografia tras el clipaje, que incluye ambas porciones del aneurisma y el anillo distal. F y G: Angiografía oblicua y lateral postoperatoria que demuestra la exclusión del aneurisma. muñón del OS. Por fuera hay una estructura fibrosa, que separa el segmento clinoideo de la ACI del IIIp y que se conoce como membrana carotido-oculomotora (MCO). Esta membrana forma una envoltura muy tenue alrededor de la ACI clinoidea y constituye el llamado collar carotídeo y su repliegue más proximal el llamado anillo proximal o membranoso (Figura 3). Desgraciadamente, estas estructuras no se han conservado adecuadamente en todos los espécimenes estudiados, apareciendo directamente la ACI y el IIIp. De la misma forma, al no estar inyectados con silicona de colores, no es posible reconocer los elementos venosos que constituyen el seno cavernoso y que se encuentran contenidos por la referida membrana carotidooculomotora. Esto produce la falsa sensación en la mayor parte de los espécimenes estudiados de que el espacio obtenido tras la clinoidectomía es muy amplio. Para movilizar la ACI es necesario seccionar el anillo distal en sus extremos medial y lateral de su continuidad anatómica con la duramadre.

El OS constituye una importante referencia anatómica, ya que por encima y medialmente pasa el IIp, por debajo y atrás gira la ACI que recorre el surco carotídeo de la cara lateral del cuerpo del esfenoides en su segmento C4 o cavernoso y también por debajo y lateralmente se rela- 
ciona con la hendidura esfenoidal o fisura orbitaria superior (Figura 3).

\section{Aneurismas paraclinoideos trans-segmentarios C5-C6}

Se han revisado los aneurismas paraclinoideos tratados quirúrgicamente entre 1996 y 2006. De un total de 16 casos, en dos de ellos el cuello se identificó en la transición entre los segmentos C5 y C6 y en ambos casos la cúpula tenía una porción clinoidea y otra subaracnoidea.

Caso 1. (Figura 4) Mujer de 60 años, sin antecedentes ni factores de riesgo salvo sobrepeso, que sufrió una cefalea brusca durmiendo. Al ingreso el GCS era de 15 puntos, sin déficits. Los estudios de imagen mostraron una hemorragia subaracnoidea y la existencia de un aneurisma paraclinoideo izquierdo en el angio-TAC con reconstrucción tridimensional. La angiografía mostraba una lesión de cuello ancho y con el saco dirigido hacia arriba, mientras que la arteria oftálmica emergía del segmento intracavernoso de la carótida. La paciente se intervino a través de una craneotomía pterional standard, con ayuda de aspiración retrógrada cervical y control de flujo con microdoppler. En la cirugía se confirmó el aneurisma de carótida interna, con el cuello ocupando el segmento C6 y extendido hacia el segmento C5 por debajo del anillo distal. Se colocó un clip angulado excluyendo el aneurisma y la paciente fue dada de alta a domicilio sin problemas.

Caso 2. (Figura 5) Mujer de 58 años, con ansiedad y grave patología sistémica asociada, concretamente anticoagulada por fibrilación auricular, hipertensión arterial e insuficiencia renal en prediálisis con reserva renal inferior al $15 \%$. Tras sufrir un evento isquémico cerebral transitorio se identificó en los estudios de imagen un aneurisma bilobulado de carótida interna derecha. La angiografía con gadolinio mostraba una lesión bilobulada dirigida hacia arriba. En la cirugía, realizada con ayuda de aspiración retrógrada y microdoppler, se demostró tras la clinoidectomía que la lesión tenía un cuello amplio y repartido entre los segmentos C5 y C6, que se excluyó con un clip angulado. La paciente fue dada de alta a domicilio con una ptosis palpebral que se resolvió completamente y se ha reintroducido la anticoagulación. La angiografía con gadolinio ha demostrado la exclusión del aneurisma.

\section{Discusión}

\section{Segmentos de /a arteria carótida interna}

A pesar de su interés anatomoclínico la nomenclatura de los segmentos de la ACI permanece confusa. La primera clasificación fue de Fischer en el año 1938 y la más reciente modificación ha sido hecha en 1996 por Bouthillier et $\mathrm{al}^{3}$. Estos últimos autores consideran la ACI dividida en las siguientes porciones: el segmento cervical $(\mathrm{Cl})$ hasta la entrada de la ACI en el canal carotídeo; segmento petroso (C2) hasta el agujero rasgado anterior o foramen lacerum; segmento lacerum (C3) hasta el inicio del ligamento petrolingual; segmento cavernoso (C4) hasta el anillo proximal; segmento clinoideo (C5) entre los anillos proximal y distal; y, finalmente, la porción intradural o cisternal, con el segmento oftálmico (C6) hasta la salida de la comunicante posterior y el segmento de la comunicante posterior (C7) hasta la bifurcación carotídea. Esta nomenclatura es importante, ya que introduce el segmento C5 o clinoideo de la ACI que se expone tras la clinoidectomía anterior. Dicho segmento de la ACI se caracteriza porque la arteria no es todavía intradural, ya que está separada del espacio subaracnoideo por el anillo distal que se continúa con la duramadre. El segmento clinoideo de la ACI se convierte en intradural gracias a la clinoidectomía anterior intradural descrita. En teoría, el segmento C5 o clinoideo tampoco sería intracavernoso, ya que la membrana carotido-oculomotora la separa de las venas del seno cavernoso $^{3,12}$. Sin embargo, habitualmente el anillo proximal no es competente y entre el collar carotídeo y la adventicia de la ACI se pueden encontrar venas dependientes del seno cavernoso, formando el llamado espacio venoso clinoideo, por lo que para algunos autores este segmento clinoideo de la ACI sería auténticamente intracavernoso ${ }^{14,15}$. Otros autores se consideran incapaces de determinar si el segmento clinoideo es intra o extracavemoso ${ }^{10}$, siendo en realidad esto poco relevante desde el punto de vista quirúrgico.

\section{Aneurismas trans-segmentarios C5-C6}

Kim et al, hacen una magnífica discusión acerca de la nomenclatura y clasificaciones de los aneurismas que asientan en la ACI cercana a la clinoides anterior o paraclinoideos ${ }^{10}$. De nuevo esta nomenclatura es muy confusa y abundan las clasificaciones. La tendencia clásica era nombrar los aneurismas con la rama arterial con la que se relacionan. Sin embargo, las ramas más importantes de la zona (oftálmica e hipofisaria superior) muestran una gran variabilidad anatómica y hay localizaciones típicas de aneurismas sin ninguna rama arterial vecina. Por ello, cada aneurisma debería identificarse indicando dónde tiene su cuello (extradural: segmentos C4 y C5; intradural: segmento (6), cúal es la proyección del saco y dónde está la cúpula (intradural o extradural). La localización del cuello y proyección son cruciales en la planificación del abordaje quirúrgico, mientras que la ubicación de la cúpula se corresponde con el riesgo de hemorragia subaracnoidea. Desgraciadamente, incluso los estudios de imagen mas precisos son incapaces de demostrar muchos de estos elemen- 
tos anatómicos, como por ejemplo, los anillos carotídeos, por lo que las auténticas relaciones del aneurisma sólo podrían definirse definitivamente en la cirugía.

Al-Rodah et al describieron en 1993 un subgrupo de aneurismas del seno cavernoso que denominan "aneurismas transicionales", y que se caracterizan porque tienen su cuello completamente en el seno cavernoso y su cúpula en el espacio subaracnoideo, por lo que tienen riesgo de sangrado subaracnoideo ${ }^{1}$. En su trabajo no hacen referencia alguna a segmentos carotídeos y sólo indican que "su cuello se localiza generalmente en la rodilla anterior de la ACI cavernosa, en la parte más distal del segmento cavernoso de la ACI'. Se trata de lesiones poco descritas, ya que es difícil con las técnicas de imagen identificar adecuadamente su cuello y los diferentes elementos anatómicos de referencia. En la serie de Iihara et al sobre 111 aneurismas paraclinoideos no rotos sólo hay 1 aneurisma transicional ${ }^{9}$. Hoh et al, sobre un total de 238 aneurismas paraclinoideos encuentran 12 lesiones (5\%) “originadas en el seno cavernoso y que se extienden al espacio subdural" 6 . Frente a estos datos, Al-Rodah et al encuentran 23 aneurismas transicionales de un total de 118 casos de aneurismas de seno cavernoso ${ }^{1}$. Más detallada es la descripción de Meyer et al, basada en hallazgos quirúrgicos, cuando define los aneurismas del segmento clinoideo, que considera extracavernoso, como aquéllos que tienen su cuello proximal al anillo distal aunque su cúpula emerja intraduralmente y que de esta forma diferencia claramente de los "aneurismas transicionales" 12 .

Los dos aneurismas descritos en nuestro trabajo son lesiones con el cuello situado en la transición entre los segmentos C5 y C6, como se ha demostrado en la cirugía y por esta razón los denominamos "trans-segmentarios C5-C6". Se trata de lesiones relativamente pequeñas con un cuello amplio que se extiende por encima y debajo del anillo distal, es decir, con una porción del cuello intradural y otra extradural. Las lesiones tenían, de la misma forma, una porción de su cúpula intradural y otra en el espacio clinoideo. Otras tres lesiones semejantes han sido descritas por Horowitz et al, White et al y Kim et al ${ }^{8,10,17}$. Cuando se trata de lesiones de tamaño suficiente es posible apreciar en la proyección angiográfica oblicua la existencia de una hendidura que, cuando es intensa, les da el aspecto de unas orejas de ratón y que sugiere la existencia de dos aneurismas, aunque la cirugía demuestra que se trata de uno solo. Horowitz et al describieron este signo angiográfico ("dural waisting sign"), que es característico de este tipo de lesiones ${ }^{8,17}$. La indentación del saco se debe al efecto de cinturón del anillo distal, por lo que corresponderían exactamente con los aneurismas transicionales de Beretta ${ }^{2}$.

Horowitz et $\mathrm{al}^{8}$ y White et $\mathrm{al}^{17}$ describen dos casos que trataron con trapping y Kim et $\mathrm{al}^{10}$ muestran un caso en una ilustración de su publicación, sin indicar el tratamiento recibido. Es difícil explicar la existencia de aneurismas en esta porción de la carótida interna, sin ramas, bifurcaciones o cambios de dirección. Lasjaunas y Berenstein ${ }^{11}$ clasifican los segmentos de la ACI según criterios embriológicos y resulta que el segmento 6-clinoideo de su clasificación se origina de la arteria aorta dorsal entre la arteria maxilar primitiva y la oftálmica dorsal, mientras que el segmento 7-terminal se origina distal a la arteria oftálmica ventral, lo que podría determinar la persistencia de puntos murales débiles en dicha transición sobre los que eventualmente se desarrollaría el aneurisma.

\section{Clinoidectomía anterior intradural}

Entre los principios básicos de seguridad de la cirugía de los aneurismas se incluye disponer del control proximal y distal de la arteria y atacar la lesión por el cuello. El control distal de los aneurismas paraclinoideos de la ACI se hace proximalmente a la salida de la arteria comunicante posterior. En cualquier lesión, el control proximal se hace con seguridad en la ACI cervical, donde se puede montar adicionalmente un sistema de aspiración retrógrada que favorece el manejo del aneurisma ${ }^{5}$. En el caso de aneurismas con cuello intradural otro punto de control proximal sería el segmento clinoideo de la ACI tras la clinoidectomía, aunque ahora no se puede realizar la aspiración retrógrada. Sin embargo, en caso de aneurismas con el cuello situado en la transición entre los segmentos C5 y C6 o en el segmento clinoideo C5 es obligatorio el control proximal cervical. En general, la exploración de cuello de cualquier aneurisma paraclinoideo y la colocación del clip se facilitan enormemente con la clinoidectomía anterior.

Sin embargo, la propia clinoidectomía exige ciertas precauciones ${ }^{16}$. En este sentido, es más segura la clinoidectomía intradural que la extradural; si se mantiene el faldón dural durante el fresado óseo se protege el nervio óptico y la cúpula del aneurisma; es más segura la resección a pequeños trozos que la resección en bloque y, finalmente, alrededor de un $10 \%$ de las clinoides están neumatizadas por prolongaciones de los senos paranasales y su resección puede seguirse de rinolicuorrea o meningitis. Además, con independencia de su origen, los aneurismas que crecen en el restringido espacio clinoideo pueden erosionar la clinoides y su resección sin control proximal de la carótida ir seguida de su ruptura catastrófica ${ }^{7}$.

\section{Bibliografía}

1. Al-Rodhan, N.R.F., Piepgras, D.G., Dundt, T.M.: Transitional cavernous aneurysms of the internal carotid artery. Neurosurgery, 1993; 33: 993-998.

2. Beretta, F.: The paraclinoid aneurysms and the distal dural ring: a new classification. J Neurol Sci, 2004; 48: 161- 
175.

3. Bouthillier, A., van Loveren, H.R., Keller, J.T.: Segments of the internal carotid artery: a new classification. Neurosurgery, 1996; 38: 425-433.

4. Dolenc, V.V.: A combined epi- and subdural direct approach to carotid-ophthalmic aneurysms. J Neurosurg, 1985; 62: 667-672.

5. González-Darder, J.M., Gil-Salú, J.L.: La "técnica de Dallas" en el tratamiento de los aneurismas de la arteria oftálmica de gran tamaño. Neurocirugía, 1999; 10: 55-60.

6. Hoh, B.L., Carter, B.S., Budzik, R.F., Putman, C.M., Ogilvy, C.S.: Results after surgical and endovascular treatment of paraclinoid aneurysms by a combined neurovascular team. Neurosurgery, 2001; 48: 78-90.

7. Horouse, K., Heros, R.C.: Subclinoid carotid aneurysm with erosion of the anterior clinoid process and fatal intraoperative rupture. Neurosurgery, 1992; 2: 356-359.

8. Horowitz, M., Fichtel, F., Samson, D., Purdy, P.: Intracavernous carotid artery aneurysms: the possible importance of angiographic dural wasting. A case report. Surg Neurol, 1983; 46: 549-552.

9. Iihara, K., Murao, K., Sakai, N., Shind, A., Sakai, H., Higashi, T., Kogure, S., Takahashi, J.C., Hayashi, K., Ishibashi, T., Nagata, I.: Unruptured paraclinoid aneurysms: a management strategy. J Neurosurg 2003; 99: 241-247.

10. Kim, J.M., Romano, A., Sanan, A., van Loveren, H.R., Keller, J.T.: Microsurgical anatomical features and nomenclature of the paraclinoid region. Neurosurgery, 2000; 46: 670682 .

11. Lasjaunas, P., Berenstein, A.: Arterial anatomy: Introduction. En: Surgical neuroangiography: functional anatomy of craniofacial arteries. Springer-Verlag, Berlin, 1987, pp 1-32.

12. Meyer, F.B., Friedman, J.A., Nichols, D.A., Windschitl, R.N.: Surgical repair of clinoidal segment carotid artery aneurysms unsuitable for endovascular treatment. Neurosurgery, 2001; 48: 476-488.

12. Parkinson, D., West, M.: Lesions of the cavernous sinus. En Youmans JR (ed): Neurological Surgery. Philadelphia, WB Saunders, 1982. pp 3004-3023.

13. Rothon, A.: The cavernous sinus, the carotid venous plexus and the carotid collar. Neurosurgery 51 (Suppl.1), 2002; 375-410.

14. Seoane, E., Rothon, Al., de Oliveira, E.: Microsurgical anatomy of the dural collar (carotid collar) and rings around the clinoid segment of the internal carotid artery. Neurosurgery, 1998; 42: 869-886.

15. Takahashi, J.A., Kawarazaki, A., Ashimoto, N.: Intradural en-bloc removal of the anterior clinoid process. Acta Neurochir (Wien), 2004; 146: 505-509.

16. White, J.A., Horowitz, M.B., Samson, D.: Dural waisting as a sign of subarachnoid extension of cavernous carotid aneurysms: a follow-up case report. Surg Neurol, 1999; 52: 607-610.

González-Darder, J.M.: Clinoidectomía anterior intradural. Estudio anatomo-clínico y utilidad en el tratamiento de los aneurismas paraclinoideos trans-segmentarios C5-C6. Neurocirugía 2007; 18: 201-208.

Correspondencia postal: José M. González-Darder. Servicio de Neurocirugía. Hospital General de Castellón. Avda de Benicassim s/n. 12003 Castellón de la Plana. 